\title{
Fatores de risco para o baixo peso ao nascer em gestantes de baixa renda
}

\section{Risk factors for low birth weight among low-income pregnant women}

\author{
Sylvia do Carmo Castro FRANCESCHINI ${ }^{1}$ \\ Sílvia Eloiza PRIORE ${ }^{1}$ \\ Nila Patrícia Freire PEQUENO² \\ Danielle Góes da SILVA ${ }^{1}$ \\ Dirce Maria SIGULEM ${ }^{2}$
}

\section{RE S U M O}

Foram analisadas, quanto à exposição a fatores de risco para o baixo peso ao nascer, 77 gestantes no último trimestre da gestação, residentes em favelas da região de Vila M ariana, município de São Paulo, acompanhadas pelo Projeto Favela, desenvolvido pela Universidade Federal de São Paulo/Escola Paulista de Medicina. As variáveis maternas de maior impacto sobre o peso ao nascer foram paridade e estatura. Primíparas geraram crianças com diferença média de peso ao nascer de $-264 \mathrm{~g}$ em relação aos recém-nascidos das multíparas. Mulheres com estaturas abaixo de $150 \mathrm{~cm}$ tiveram crianças com diferença média de peso ao nascer de $-287 \mathrm{~g}$ em comparação com os neonatos de mães com estaturas maiores. A penas $2,6 \%$ das crianças nasceram com baixo peso, apesar da alta exposição da população estudada aos fatores de risco.

Termos de indexação: gravidez, peso ao nascer, estado nutricional, riscos gestacionais, intervenção nutricional.

\section{A B S T R A C T}

This paper assessed 77 pregnant women undergoing the last three months of gestation, in order to analyze their exposure to risk factors for low birth weight. They lived in slums in the region of Vila Mariana, a district

\footnotetext{
${ }^{1}$ Departamento de Nutrição e Saúde, Universidade Federal de Viçosa. Campus Universitário, 36571-000, Viçosa, MG, Brasil. Correspondência para/Correspondence to: S.C.C.FRANCESCHINI.E-mail: sylvia@ufv.br

2 Escola Paulista de Medicina, Universidade Federal de São Paulo.
} 
in the city of São Paulo, and were followed up by the "Projeto Favela", developed by the Federal University of São Paulo/School of Medicine. The maternal variables which most influenced birth weight were the mother's number of pregnancies and height. Primiparous women gave birth to newborns with lower birthweight, presenting an average difference of $-264 \mathrm{~g}$ when compared with infants born to multiparous mothers. Women whose height did not exceed $150 \mathrm{~cm}$ also delivered infants with low er birth weight, demonstrating an average difference of $-287 \mathrm{~g}$ when compared with neonates born to taller mothers. Only $2.6 \%$ of the infants presented low birth weight, despite the high exposure of the studied population to risk factors.

Index terms: pregnancy, birth weight, nutritional status, gestational risks, nutritional intervention.

\section{N T R O D U Ç Ã O}

Dentre os indicadores de saúde eleitos para predizer a qualidade de vida da população, um dos mais importantes é o peso ao nascer, especialmente porque diz respeito à saúde reprodutiva das mulheres.

0 peso ao nascer reflete a qualidade da atenção dispensada à gestante, seu estado nutricional antes e durante a gestação e os fatores de risco aos quais está exposta, permitindo a identificação de áreas e situações de risco e 0 direcionamento de políticas e programas específicos de saúde e nutrição ${ }^{1}$.

Segundo a Pan American Health Organization (PAHO) (1991)², há evidências de que o peso do recém-nascido, do qual depende sua saúde, seu crescimento e seu desenvolvimento posteriores, é intimamente dependente do estado nutricional materno.

Conforme relataram Puffer \& Serrano $(1988)^{3}$ ao estudarem as características do peso ao nascer de crianças da América Central, com dados obtidos em diversos países, os dois mais importantes fatores relacionados ao baixo peso ao nascer são o peso pré-gestacional e o ganho de peso na gestação. Além disso, os programas que têm como finalidade melhorar o estado nutricional das mães, tanto antes quanto durante a gestação, são eficazes na redução da mortalidade infantil pelo seu impacto sobre o peso ao nascer.

0 peso pré-gestacional avalia o risco inicial de um prognóstico desfavorável da gestação, determina o ganho de peso recomendado e direciona intervenções nutricionais. Peso pré-gestacional inadequado, acompanhado por ganho de peso insuficiente, aumenta o risco de baixo peso ao nascer e de mortalidade perinatal, neonatal e infantili1,2,4.

O Índice de M assa Corporal (IMC), o qual proporciona informações com relação às reservas energéticas, tem sido utilizado com freqüência para determinar e monitorar o ganho de peso baseado no estado nutricional pré-gravídico ${ }^{2,4}$. De acordo com recomendações da National Academy of Sciences $(1990)^{5}$, mulheres com baixo peso pré-gestacional (IMC $\leq 19,8)$, devem ter um ganho de peso total na gestação de 12,5 a $18,0 \mathrm{~kg}$. As que entram na gestação com peso normal (IMC de 19,8 a 26,0), sobrepeso (IMC de 26,0 a 29,0 ) e obesidade (IMC $>29,0$ ) devem ganhar, respectivamente, de 11,5 a 16,0, de 7,0 a 11,5 e o mínimo de $6,0 \mathrm{~kg}$. Abrams et al. $(2000)^{6}$, reavaliando a proposta da National Academy of Sciences, destacam que esses valores de ganho de peso gestacional estão relacionados a melhores condições de saúde da mãe e de peso ao nascer do concepto.

A estatura, como outras medidas antropométricas, tem sido utilizada para se avaliar os riscos de baixo peso ao nascer, mortalidade perinatal, neonatal e infantil e performance lactacional. Os pontos de corte que predizem o baixo peso ao nascer se situam entre $140 \mathrm{e}$ $150(\mathrm{~cm})^{2}$. Siqueira et al. $(1975)^{7}$ encontraram em mulheres brasileiras com estaturas inferiores a $150 \mathrm{~cm}$ maiores probabilidades de gerarem crianças com baixo peso ao nascer. 
A Curva de Rosso $(1985)^{8}$ tem como objetivo avaliar o ganho de peso baseado na avaliação do peso para estatura de acordo com a idade gestacional. Esta curva possibilita o diagnóstico nutricional na primeira consulta e o monitoramento dos ganhos ponderais subseqüentes, além de apresentar a vantagem de ser um instrumento de fácil execução e baixo custo. Destaca-se por ter sido, até o momento, a única curva validada para a população brasileira ${ }^{9}$.

Dentre os principais determinantes do peso ao nascer incluem-se, além daqueles relacionados ao estado nutricional materno, a idade materna, a paridade, a atenção pré-natal, o tabagismo e a história reprodutiva ${ }^{1,10-12}$. Estes são condicionados pelo nível socioeconômico da família ${ }^{12,13}$.

Com relação à idade, sabe-se que mães muito jovens são fisiologicamente imaturas para suportar os estresses da gravidez e, conforme referenciado por Story $(1990)^{14}$, o risco é especialmente maior quando a gestação acontece em menos de dois anos após a menarca. A gestante adolescente tem menores ganhos de peso e é questionado se ela compete com o feto pelos nutrientes, em prol do seu próprio crescimento ${ }^{15}$.

A paridade e o intervalo interpartal implicam em riscos de diferentes magnitudes para a mulher e o neonato. As primíparas têm normalmente crianças com média de peso ao nascer inferiores às multíparas ${ }^{16}$. No entanto, grandes multíparas, têm maior tendência a intervalos interpartais mais curtos, importante fator de risco para o baixo peso ao nascer, prematuridade, mortalidade neonatal e infantil e desnutrição na infância ${ }^{17}$.

0 Projeto Favela, desenvolvido pela Universidade Federal de São Paulo/Escola Paulista de M edicina, teve início no final da década de 80 e caracteriza-se como um modelo de atenção primária à saúde da população materno-infantil residente em favelas da região de Vila Mariana, São Paulo.

Inicialmente voltado para a redução da desnutrição energético-proteíca em crianças menores de cinco anos, através de atendimento com equipe multiprofissional nos domicílios, e para divulgação das ações básicas de saúde, a partir de 1993 este Projeto expandiu as atividades para gestantes e nutrizes, através de trabalhos com grupos de mães e da intervenção nutricional individualizada.

Este trabalho tem como objetivo caracterizar as condições gestacionais e sua relação com o peso ao nascer, em uma amostra de mulheres de baixo nível socioeconômico, residentes em favelas da região de Vila M ariana, município de São Paulo, que receberam intervenção nutricional através do Projeto Favela, da Universidade Federal de São Paulo/Escola Paulista de Medicina.

\section{CASUÍSTICA E M ÉTODOS}

0 estudo foi desenvolvido com 77 gestantes de baixo nível socioeconômico, com idade variando de 15 a 48 anos, sendo a mediana de 23 anos, residentes em quatro favelas da região de Vila Mariana, município de São Paulo.

Nas comunidades estudadas foram identificadas 124 gestantes, das quais $47(38,0 \%)$ foram excluídas por motivos de mudança ou desistência. A perda amostral, em estudos prospectivos com populações residentes em favelas, é freqüentemente grande em virtude da intensa mobilidade.

Ao ser identificada, independentemente da idade gestacional, a gestante era convidada a participar dos grupos de mães organizados pela equipe do Projeto Favela, e recebia orientações mensais sobre um dos seguintes temas: alimentação da gestante, importância do acompanhamento pré-natal, ganho de peso na gestação, anemia, tabagismo, etilismo, hipertensão arterial, aleitamento materno, cuidados com o recém-nascido e outros.

A visita domiciliar às gestantes foi realizada no início do terceiro trimestre gestacional, quando aplicou-se um formulário para avaliar a história 
obstétrica, o acompanhamento pré-natal e a presença de tabagismo.

Para avaliação antropométrica, realizou-se pesagem da gestante, utilizando balança eletrônica, com capacidade de $150 \mathrm{~kg}$ e divisão de $50 \mathrm{~g}$. A estatura foi verificada através de fita métrica metálica, com extensão de 2 metros, dividida em centímetros e subdividida em milímetros. Para realização das medidas foram utilizadas as técnicas preconizadas por Jelliffe $(1968)^{18}$.

A gestante recebeu orientação nutricional de acordo com o IMC pré-gestacional ${ }^{10}, 0$ critério de Rosso 4 e a adequação da dieta foi avaliada pelo Recordatório de 24 horas.

No décimo dia após o parto foram coletadas informações com relação ao tipo de parto e peso ao nascer do concepto; foi efetuado ainda o cálculo do intervalo interpartal do penúltimo para o último filho. 0 ganho de peso total na gestação foi calculado com base no peso pré-gestacional recordado e no obtido durante a última consulta pré-natal.

Considerou-se como gestantes pertencentes ao grupo de risco as que apresentavam: idade inferior a 20 anos e superior a 35 anos, intervalo interpartal inferior a 24 meses, paridade maior ou igual a 5 e tabagismo durante a gestação ${ }^{17,19}$. Gestantes em risco nutricional foram acompanhadas pela equipe do Projeto Favela até o final da gravidez.

Para avaliação do estado nutricional da gestante, foram utilizados os seguintes critérios: estatura inferior a $150 \mathrm{~cm}$, IMC pré-gestacional igual ou inferior a 19,8 e superior a 26,0 , ganho de peso na gestação menor ou igual a $7,0 \mathrm{~kg}$ e superior a 16,0kg e baixo peso, sobrepeso e/ou obesidade de acordo com o critério de Rosso ${ }^{2,5,8}$.

Para verificar possíveis diferenças das médias de peso ao nascer com relação às variáveis e à antropometria materna, aplicou-se o teste " $\mathrm{t}$ " para médias independentes (para dois grupos), e a análise de variância para médias independentes (para três ou mais grupos). Estabeleceu-se como nível de rejeição para a hipótese de nulidade valores de $p$ inferiores a 0,05 . Os resultados estatisticamente significantes foram assinalados com $(*)$.

\section{RESULTA D OSE DISCUSSÃ O}

A idade mediana de 23 anos das gestantes foi considerada adequada para reprodução, uma vez que nesta fase se encontram os menores riscos para a performance fetal e obstétrica. No entanto, $27,3 \%$ e 3,9\% apresentavam idade inferior a 20 e superior ou igual a 35 anos, respectivamente, totalizando 24 gestantes $(31,2 \%$ ) com fatores de riscos inerentes à idade (Tabela 1). Embora não apresentando significância, provavelmente em conseqüência do tamanho amostral e da variabilidade $(p=0,08)$, as adolescentes geraram crianças com diferença média de peso ao nascer de-184g em comparação com os recém-nascidos de mulheres com idade ideal para reprodução de 20 a 35 anos, enquanto esta diferença foi de $246 \mathrm{~g}$ para gestantes com idade superior a 35 anos.

Na gestação durante a adolescência, os fatores biológicos (imaturidade do sistema reprodutivo, ganho de peso gestacional insuficiente), somados aos fatores socioculturais (baixa escolaridade, pobreza e marginalidade social), e a alimentação estariam envolvidos na ocorrência do baixo peso ao nascer ${ }^{10}$. Considerando a alta freqüência de gestação na adolescência observada nesta população, bem como a imaturidade fisiológica destas gestantes e o fato de pertencerem ao baixo nível socioeconômico, verifica-se a necessidade de trabalho de orientação voltado para este grupo.

Com relação à paridade, $37,7 \%$ e 62,3\% das gestantes eram primíparas e multíparas, respectivamente (Tabela 1 ). A diferença média do peso ao nascer dos recém-nascidos de mães primíparas foi de $-264 \mathrm{~g}$ em relação aos neonatos das multíparas $(p=0,002)$. Esta diferença se manteve quando o fator idade materna foi controlado. 
Tabela 1. Peso ao nascer segundo características maternas relacionadas à gestação e ao parto.

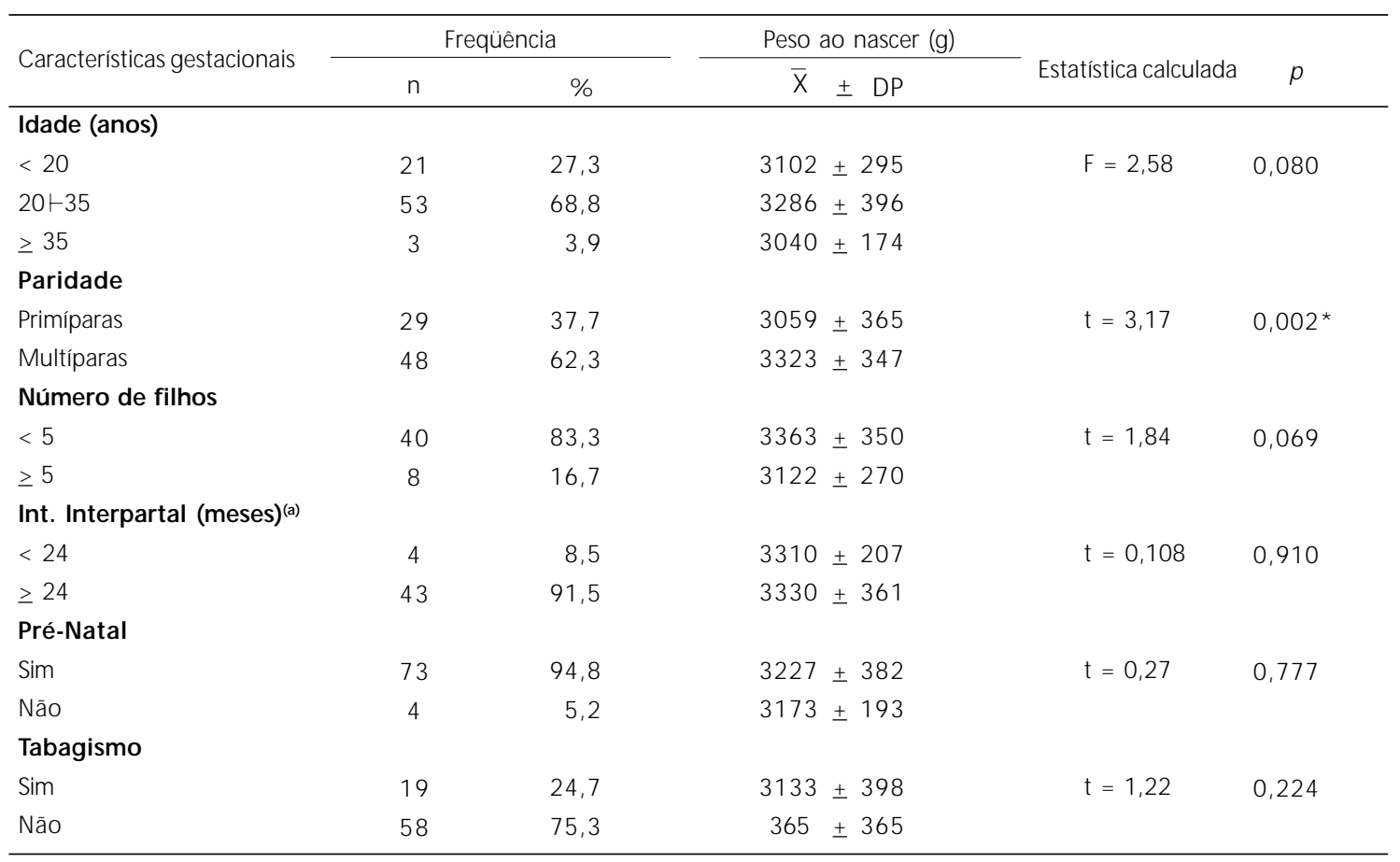

(a) 1 - sem informação.

Entre as multíparas, 8 (16,7\%) apresentavam riscos em virtude do grande número de gestações (Tabela 1). A diferença entre as médias de peso ao nascer foi de $-241 \mathrm{~g}$ entre mulheres com cinco ou mais gestações, e, apesar de as diferenças não terem sido estatisticamente significantes ( $p=0,069$ ), podem ser biologicamente significativas. 0 efeito da freqüência dos ciclos reprodutivos sobre as reservas nutricionais maternas, determinando a "síndrome de depleção materna" ${ }^{20}$, deve ser motivo de preocupação em comunidades carentes, onde a disponibilidade de alimentos é freqüentemente escassa, podendo agravar o estado nutricional materno, com esgotamento das reservas corporais.

Como pode ser observado (Tabela 1), não se alcançou diferença estatisticamente significante entre as médias de peso ao nascer para intervalo interpartal ( $p=0,910)$; no entanto, 0 resultado deve ser analisado com cuidado, tendo em vista o pequeno tamanho amostral do grupo considerado de risco para este fator.
O acompanhamento pré-natal exerce efeito protetor com relação ao baixo peso ao nascer ${ }^{10,11}$. Das gestantes estudadas nas favelas de Vila Mariana, 94,8\% fizeram o pré-natal (Tabela 1). Tomando-se como referência os dados da Pesquisa Nacional sobre Demografia e Saúde $(1997)^{21}$, os quais relatam uma cobertura pré-natal de $51,1 \%$ em termos nacionais e de $60,6 \%$ para o município de São Paulo, poder-se-ia concluir, então, que esta atenção é satisfatória para a população estudada. Entretanto, considerando-se a proximidade destas favelas aos serviços básicos de saúde e ao Hospital São Paulo, a exclusão de $5,2 \%$ pode indicar dificuldade de acesso, dessas mulheres, à assistência pré-natal.

A ausência do acompanhamento pré-natal e o hábito de fumar da mãe são geralmente associados com o baixo peso ao nascer ${ }^{11,12}$. Neste estudo, as gestantes que não receberam atenção pré-natal e as tabagistas geraram crianças com médias de peso ao nascer inferiores, mas as diferenças não foram estatisticamente signifi- 
cantes ( $p=0,777$ e $p=0,224$, respectivamente). Para a ausência de pré-natal, o tamanho amostral pode ter interferido no resultado encontrado. Quanto ao tabagismo, a presença ou ausência não se mostrou como fator discriminador entre os grupos, e talvez melhor avaliação possa ser obtida considerando-se o número de cigarros por dia e sua duração no decorrer da gestação.

Conforme se observou na análise dos dados antropométricos maternos, $31,1 \%$ e $13,5 \%$ das mulheres estudadas iniciaram a gestação com baixo peso e sobrepeso/obesidade, respectivamente, totalizando $44,6 \%$ com riscos inerentes ao estado nutricional pré-gravídico, expresso em IMC pré-gestacional (Tabela 2). A diferença entre as médias de peso ao nascer aumenta com 0 incremento da adequação de peso pré-gestacional/estatura. M ulheres que iniciaram a gestação com baixo peso geraram crianças com médias de peso ao nascer apresentando diferenças de $-60 \mathrm{~g} \mathrm{e}-300 \mathrm{~g}$, respectivamente, em relação aos pesos obtidos entre as eutróficas e as com sobrepeso/obesidade. No entanto, as diferenças não foram estatisticamente significantes $(p=0,122)$. A pesar deste achado, é indiscutível a importância deste indicador na avaliação do estado nutricional de gestantes, pois seguindo orientação da PAHO (1991)² para análise conjunta do IM C pré-gestacional e ganho de peso durante a gestação, mulheres com baixo peso no início da gestação ganharam em média $13,4 \mathrm{~kg}$, enquanto o ganho ponderal foi de 10,3 e 10,7 kg, respectivamente, para as eutróficas e as com sobrepeso ou obesidade.

Ainda analisando o ganho de peso na gestação (Tabela 2), com o aumento deste houve incremento nas médias de peso ao nascer, excetuando-se o grupo cujo ganho de peso foi excessivo. Lizo et al. (1998) ${ }^{13}$ também não encontraram correlação entre ganho de peso gestacional superior a $16 \mathrm{~kg}$ e incremento ponderal do recém-nascido. No presente estudo, ocorreu maior incremento ponderal entre os recém-nascidos de mães que ganharam entre 7,0 e 16kg na gestação.

De acordo com o critério de Rosso, 38,8\% e $16,4 \%$ das gestantes apresentavam-se com baixo peso e sobrepeso/obesidade, respectivamente, perfazendo $55,2 \%$ com riscos nutricionais no último trimestre da gestação (Tabela 2). 0

Tabela 2. Peso ao nascer segundo antropometria materna relacionadas ao período pré-gestacional e gestacional.

\begin{tabular}{|c|c|c|c|c|c|}
\hline \multirow{2}{*}{ Antropometria } & \multicolumn{2}{|c|}{ Freqüência } & Peso ao nascer $(\mathrm{g})$ & \multirow[b]{2}{*}{ Estatística calculada } & \multirow[b]{2}{*}{$\mathrm{p}$} \\
\hline & $\mathrm{n}$ & $\%$ & $\bar{X} \pm D P$ & & \\
\hline \multicolumn{6}{|l|}{ IMC Pré-gestacional (a) } \\
\hline$\leq 19,8$ & 23 & 31,1 & $3140 \pm 363$ & $F=2,16$ & 0,122 \\
\hline $19,8 \dashv 26,0$ & 41 & 55,4 & $3200 \pm 421$ & & \\
\hline$>26,0$ & 10 & 13,5 & $3440 \pm 289$ & & \\
\hline \multicolumn{6}{|c|}{ Ganho de peso na gestação $(\mathbf{K g})^{(\mathbf{b})}$} \\
\hline$\leq 7,0$ & 13 & 18,1 & $3030 \pm 537$ & $F=1,90$ & 0,137 \\
\hline $7,0 \dashv 11,5$ & 26 & 36,1 & $3240 \pm 353$ & & \\
\hline $11,5 \dashv 16,0$ & 24 & 33,3 & $3330 \pm 337$ & & \\
\hline$>16,0$ & 9 & 12,5 & $3240 \pm 169$ & & \\
\hline \multicolumn{6}{|l|}{ Avaliação por Rosso ${ }^{(c)}$} \\
\hline Baixo Peso & 26 & 38,8 & $3287 \pm 320$ & $F=2,70$ & 0,072 \\
\hline Eutróficas & 30 & 44,8 & $3140 \pm 413$ & & \\
\hline Sobrepeso/Obesidade & 11 & 16,4 & $3420 \pm 308$ & & \\
\hline \multicolumn{6}{|l|}{ Estatura $(\mathrm{cm})$} \\
\hline$<150$ & 14 & 18,2 & $2989 \pm 403$ & $t=2,70$ & $0,008 *$ \\
\hline$\geq 150$ & 63 & 81,8 & $3276 \pm 350$ & & \\
\hline
\end{tabular}

(a) = 3 sem informação; (b) = 5 sem informação; (c) = 10 sem informação. 
critério de avaliação segundo Rosso (1991) ${ }^{4}$ não foi capaz de predizer o baixo peso ao nascer na população estudada $(p=0,072)$. Taddei et al. $(1991)^{9}$ recomendam que, na prática da assistência pré-natal, este instrumento deve ser utilizado em conjunto com outros parâmetros de avaliação nutricional.

Quanto à estatura, em 18,2\% das gestantes ela era inferior a $150 \mathrm{~cm}$ (Tabela 2). Este indicador foi o principal determinante para o baixo peso ao nascer. A diferença nas médias de peso ao nascer dos recém-nascidos de mães com estatura abaixo de $150 \mathrm{~cm}$ foi de $-287 \mathrm{~g}$ em comparação com o outro grupo $(p=0,008)$. Esta relação entre peso ao nascer e estatura materna é também discutida por outros autores ${ }^{1,7}$. É importante destacar que a estatura materna, além de exercer impacto sobre o peso ao nascer, tem sido utilizada para avaliar os riscos de mortalidade perinatal, neonatal e infantil e a performance lactacional2.

Com relação ao tipo de parto, foi encontrada freqüência de $36,0 \%$ de cesariana e $64,0 \%$ de parto normal. Segundo a Pesquisa Nacional sobre Demografia e Saúde (1997)21, a proporção de cesáreas no atendimento ao parto é de $36,4 \%$ para o Brasil como um todo e de $52,1 \%$ para o município de São Paulo. Se cada semana gestacional, aos nove meses, representa um acréscimo de aproximadamente $225 \mathrm{~g}$ ao peso do recém-nascido ${ }^{22}$, os partos cirúrgicos têm impacto importante, especialmente em populações de baixa renda. Estudo realizado por Ribeiro et al. $(2000)^{23}$ com duas coortes de mães adolescentes do município de Ribeirão Preto demonstrou ser o aumento do número de cesarianas um dos fatores responsáveis pela elevação do baixo peso ao nascer neste município.

A média do peso ao nascer entre os lactentes das favelas da região de Vila Mariana foi de $3223,8 \pm 374,5 \mathrm{~g}$, valor superior ao relatado por M onteiro et al. (2000)12 para o município de São Paulo (3160g). Entretanto, 22,1\% deles nasceram com peso deficiente ou insuficiente (Tabela 3), o que é fator de preocupação para a sobrevivência da criança em ambiente com condições adversas. Taddei et al. $(1991)^{9}$, em estudo com 1739 gestantes de 5 Estados brasileiros, encontrou 22,0\% de peso insuficiente, valor semelhante ao obtido nesta pesquisa.

Tabela 3. Peso de nascimento dos recém-nascidos das favelas da região de Vila Mariana, São Paulo.

\begin{tabular}{lcc}
\hline \multirow{2}{*}{ Peso ao nascer $(\mathrm{g})$} & \multicolumn{2}{c}{ Freqüência } \\
\cline { 2 - 3 } & $\mathrm{n}$ & $\%$ \\
\hline$<2500$ & 2 & 2,6 \\
$2500-3000$ & 17 & 22,1 \\
$\geq 3000$ & 58 & 75,3 \\
\hline Total & 77 & 100,0 \\
\hline
\end{tabular}

A alta incidência de recém-nascidos com peso insuficiente deve servir de alerta, pois a mortalidade neonatal precoce, neste grupo, é duas a três vezes superior à encontrada para os neonatos que nasceram com peso entre $3,5 \mathrm{e}$ $4,0 \mathrm{~kg}^{3}$.

Segundo relatou Sigulem $(1981)^{24}$, ao estudar as condições de saúde e nutrição de lactentes do município de São Paulo, no grupo de nascidos com peso deficiente ocorreram casos mais graves e maior freqüência de formas crônicas de desnutrição, em comparação com o grupo de lactentes nascidos com peso normal, reforçando a importância deste componente na sobrevida da criança.

A freqüência de baixo peso ao nascer, na amostra estudada, foi de 2,6\% (Tabela 3). Considerando os fatores de risco maternos, anteriormente observados, poder-se-ia esperar incidência mais elevada de baixo peso ao nascer no grupo estudado. A análise de tendência secular do peso ao nascer no município de São Paulo, realizada por M onteiro et al. $(2000)^{12}$, demonstrou incidência de $8,9 \%$ de baixo peso ao nascer para o município, em 1998. Desta forma, mesmo na ausência de variáveis para identificar diretamente o impacto da atenção da equipe do Projeto Favela, no último trimestre de gestação, acredita-se que esta pode ter contribuído para a menor prevalência 
de baixo peso nesta população, apesar da alta exposição a fatores de risco e da adversidade do meio ambiente.

\section{O N C L U S Ã O}

A alta exposição da população estudada aos fatores de risco não exerceu impacto sobre 0 peso ao nascer, pois observou-se reduzida prevalência de baixo peso. No entanto, como o trabalho de orientação domiciliar é a única característica diferencial de outras populações onde esta prevalência é elevada, supõe-se ter havido alguma influência deste trabalho nos resultados obtidos. Acredita-se que a valorização da mãe e do seu concepto, o alerta para os fatores de risco, a orientação nutricional e, especialmente, o compartilhamento de suas angústias e ansiedades podem modificar conceitos pré-estabelecidos e gerar maior preocupação com sua saúde. Estas considerações necessitam de maior atenção no momento do planejamento de políticas públicas de saúde e nutrição.

\section{REFERÊ N C I A S}

1. Barros FC, Victora CG, Vaughan JP, Estanislau HJ. Bajo peso al nascer en el municipio de Pelotas, Brasil: factores de riesgo. Bol Ofic Sanit Panam 1987; 102(6):541-54.

2. Pan American Health Organization. Maternal Nutrition and Pregnancy Outcomes: anthropometric assessment. Washington DC: PAHO; 1991. (Scientific Publication n.529).

3. Puffer RR, Serrano CV. Características del peso al nascer. Washington DC: OMS; 1988. (Publicación Científica, n.504).

4. Rosso P. Maternal anthropometry in prenatal care: a new maternal weight gain chart. Human Resources Division. Washington DC: The World Bank; 1991. LATHR n.21.

5. National Academy of Sciences. Nutrition during pregnancy. Part I. Weight Gain. Part II. Nutrient
Supplements. Washington DC: National Academy Press; 1990.

6. Abrams B, Altman SL, Pickett KE. Pregnancy weight gain: still controversial. Am J Clin Nutr 2000; 71(Suppl 1):1233-41.

7. Siqueira $A A F$, et al. Influência da altura e ganho de peso maternos e da idade gestacional sobre o peso do recém-nascido: estudo de 3 grupos de gestantes normais. Rev Saúde Pública 1975; 9:331-42.

8. Rosso P. A new chart to monitor weight gain during pregnancy. Am J Clin Nutr 1985; 41(3):644-52.

9. Taddei JAAC, et al. $O$ percentual do peso para altura da gestante no prognóstico do baixo peso ao nascer: estudo multicêntrico. Rev Bras Ginecol Obstet 1991; 2:78-82.

10. Gama SGN, Szw arcwald CL, Leal MC, Theme Filha M M. Gravidez na adolescência como fator de risco para o baixo peso ao nascer no município do Rio de Janeiro, 1996 a 1998. Rev Saúde Pública 2001; 35(1):74-80.

11. Mariotoni GGB, Barros Filho AAB. A gravidez na adolescência é fator de risco para o baixo peso ao nascer? J Pediatr 1998; 74(2):107-13.

12. Monteiro CA, Benicio MHA, Ortiz LP. Tendência secular do peso ao nascer na cidade de São Paulo (1976-1998). Rev Saúde Pública 2000; 34(Suppl 6):26-40.

13. Lizo CLP, Azevedo-Lizo Z, Aronson E, Segre CAM . Relação entre ganho de peso materno e peso do recém-nascido. J Pediatr 1998; 74(2):114-18.

14. Story M ED. Nutrition management of the pregnant adolescent: a practical reference guide. Washington DC: National Clearinghouse; 1990.

15. Scholl T, Hediger M L, Ances IG. Maternal growth during pregnancy and decreased infant birth weight. Am J Clin Nutr 1990; 51(5):790-3.

16. Seidman DS, Ever-Hadani P, Gale R. The effect of maternal weight gain in pregnancy on birth weight. Obstet Gynecol 1989; 74(2):240-6.

17. Huttly SRA, Victora CG, Barros FC, Vaughan JP. Birth spacing and child health in urban Brazilian children. Pediatrics 1992; 89:1049-54. 
18. Jelliffe DB. Evaluación del estado de nutrición de la comunidad. Ginebra: OM S; 1968.

19. National Research Council. Anticoncepción y Reproducción: consecuencias para la salud de mujeres y niños en el mundo en desarrolo. Buenos Aires: OPS; 1990.

20. Merchant K, Martorell R. Frequent reproductive cycling: does it lead to nutritional depletion of mothers? Prog Food Nutr Sci 1988; 12(4): 339-69.

21. Sociedade Civil Bem-Estar Familiar no Brasil. Pesquisa Nacional sobre Demografia e Saúde 1996. Rio de Janeiro: Bemfam; 1997.
22. Winikoff $\mathrm{B}$, Debrovner $\mathrm{CH}$. Anthropometric determinants of birth weight. Obstet Gynecol 1981; 58(6):678-84.

23. Ribeiro ERO, Barbieri MA, Bettiol H, Silva AAM. Comparação entre duas coortes de mães adolescentes em município do Sudeste do Brasil. Rev Saúde Pública 2000; 34(2):136-42.

24. Sigulem DM. Condições de saúde e nutrição de lactentes do município de São Paulo [tese]. São Paulo: Escola Paulista de M edicina, Unifesp; 1981.

Recebido para publicação em 28 de setembro de 2001 e aceito em 13 de agosto de 2002 